\title{
Research on Gear Decision Technology for Pure Electric Bus based on the Operating Routes
}

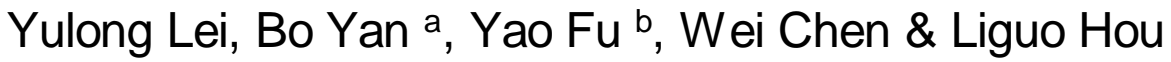 \\ Jilin University, State Key Laboratory of Automobile Simulation and Control, Changchun 130022, \\ China
}

a18843102529@163.com, bfu_yao@jlu.edu.cn

Keywords: Pure Electric Bus, Kaltman Filter, AMT.

\begin{abstract}
In order to make full use of the pure electric bus fixed route characteristic, this paper analyzes the correction algorithm of dynamic vehicle location that based on self-adaptive Kaltman filter. A method of curve and ramp recognition based on vehicle driving position and line features is proposed and ultimately determines the comprehensive shift strategy of the pure electric bus that based on fixed routes. In order to verify the correctness of the control strategy, the authors develop a pure electric vehicle with the AMT, and conduct the real vehicle verification experiment. Experimental results show that the control strategy can well identify the corners and ramps, and avoid the cycle shift, and finally improve the ride comfort of the vehicle.
\end{abstract}

\section{Introduction}

In paper [1], the author designed the powertrains parameters for the two-gear pure electric vehicle, and developed a shift schedule, but there is no research on shift schedule in specific working conditions [1]. In paper [2], the author analyzed the causes of shift cycle in conventional internal combustion engine vehicle with AMT, and developed appropriate strategies to eliminate the shift cycle [2]. In paper [3], the author proposed double hypothesis filtering algorithm for Car GPS positioning method, which could accurately locate the vehicle, but there is no vehicle positioning information used in the vehicle's shift schedule [3]. In paper [4], the author the author studied the shift schedule under various working conditions, but condition recognition model is complex, and the accuracy is not high [4].

In this paper, we research the pure electric bus with AMT which possess characteristics of fixed line, and get the GPS information through CAN bus [5]. Then we use Kalman filter to process the obtained signal that could accurately identify the vehicle location [6]. Then, we identify the typical working conditions of bus routes. Then according to the analysis of the curve and slope typical conditions, we study the shift cycle appears in two conditions, and develop the appropriate shift strategy. Finally we performed real vehicle experiments to test shift strategy.

\section{The vehicles dynamic position error correction based on self-adaptive Kalman filter}

Kalman filtering technique is a very sophisticated filtering technology, which is a continuous forecasting, constantly revised procedures, and can to reduce the position error of vehicle. Moreover, this method is implemented in software methods, no additional cost.

1. Establish the system equation

Firstly, the state variable is selected to determine the position, velocity and acceleration of the vehicle. The selected state variables are shown as follow:

$$
X=\left[\begin{array}{llllllllllll}
x & v_{x} & a_{x} & \varepsilon_{x} & y & v_{y} & a_{y} & \varepsilon_{y} & z & v_{z} & a_{z} & \varepsilon_{z}
\end{array}\right]^{T}
$$

In formula (1), $x, v_{x}, a_{x}, \varepsilon_{x}$ respectively represent the position, velocity, acceleration and position error in the $\mathrm{x}$ direction. $y, v_{y}, a_{y}, \varepsilon_{y}$ respectively represent the position, velocity, acceleration and position error in the y direction. $z, v_{z}, a_{z}, \varepsilon_{z}$ respectively represent the position, velocity, acceleration and position error in the $\mathrm{z}$ direction. The GPS positioning total position error in all directions are 
equivalent to the sum of colored noise and average value that according with the first-order markov. From this, we can get the state equation of the system:

$$
\dot{X}(t)=A X(t)+U(t)+W(t)
$$

In formula (2), $A$ is the state matrix of the system.

$$
\begin{aligned}
A & =\left[\begin{array}{ccc}
A_{x} & 0_{4 \times 4} & 0_{4 \times 4} \\
0_{4 \times 4} & A_{y} & 0_{4 \times 4} \\
0_{4 \times 4} & 0_{4 \times 4} & A_{z}
\end{array}\right] \\
A_{x} & =\left[\begin{array}{cccc}
0 & 1 & 0 & 0 \\
0 & 0 & 1 & 0 \\
0 & 0 & -\frac{1}{\tau_{a x}} & 0 \\
0 & 0 & 0 & -\frac{1}{\tau_{x}}
\end{array}\right] \\
A_{y} & =\left[\begin{array}{cccc}
0 & 1 & 0 & 0 \\
0 & 0 & 1 & 0 \\
0 & 0 & -\frac{1}{\tau_{a y}} & 0 \\
0 & 0 & 0 & -\frac{1}{\tau_{y}}
\end{array}\right] \\
A_{z} & =\left[\begin{array}{cccc}
0 & 1 & 0 & 0 \\
0 & 0 & 1 & 0 \\
0 & 0 & -\frac{1}{\tau_{a z}} & 0 \\
0 & 0 & 0 & -\frac{1}{\tau_{z}}
\end{array}\right]
\end{aligned}
$$

$U(t)$ is the current average.

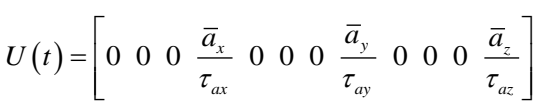

$W(t)$ is the system noise.

$W(t)=\left[\begin{array}{llllllllllll}0 & 0 & \omega_{a x} & \omega_{x} & 0 & 0 & \omega_{a y} & \omega_{y} & 0 & 0 & \omega_{a z} & \omega_{z}\end{array}\right]$

Above all, $\tau_{a x}, \tau_{a y}, \tau_{a z}$ respectively represent the time constant of acceleration. $\tau_{x}, \tau_{y}, \tau_{z}$ respectively represent the time constant in first-order Markov process. $\bar{a}_{x}, \bar{a}_{y}, \bar{a}_{z}$ respectively represent the mean acceleration. $\omega_{a x}, \omega_{a y}, \omega_{a z}$ respectively represent gaussian white noise in $\left(0, \sigma_{a x}^{2}\right),\left(0, \sigma_{a y}^{2}\right)$, $\left(0, \sigma_{a z}^{2}\right)$.

2. Establish the observation equation

We assume that $Z=L=\left[\begin{array}{lll}L_{x} & L_{y} & L_{z}\end{array}\right]^{T}$, in formula, $L_{x}, L_{y}, L_{z}$ are the measured value of the locating results. So we can get the observation equation that show as fllow:

$Z=H X+V$

In formula (9), $\mathrm{V}$ is the observation noise vector, $\mathrm{H}$ is observation equation.

3.Establish the adaptive kalman filter equation

Firstly, identify discrete adaptive Kalman filter equations:

$$
\begin{aligned}
& X(k+1)=\phi_{1}(k+1, k) X(k) \\
& X(k+1)=X(k+1, k)+K(k+1) * \\
& {[Z(k+1)-H(k+1) X(k+1, k)]} \\
& K(k+1)=P(k+1, k) H^{T}(k+1) * \\
& {\left[H(k+1) P(k+1) H^{T}(k+1)+R(k+1)\right]^{-1}}
\end{aligned}
$$




$$
\begin{aligned}
& P(k+1, k)=\lambda(k+1) \phi(k+1, k) * \\
& P(k) \phi^{T}(k+1, k)+Q(k) \\
& P(k+1)=[I-K(k+1) H(k+1)] P(k+1, k)
\end{aligned}
$$

The determination method of $\bar{a}_{x}$ is show in formula (6), the determination method of $\sigma_{a x}^{2}$ is show in formula (7).

$$
\begin{aligned}
& \bar{a}_{x}(k+1)=\bar{a}_{x}(k+1 / k) \\
& \sigma_{a x}^{2}= \begin{cases}\frac{4-\pi}{\pi}\left[a_{\text {max }}-\hat{a}(k)\right]^{2} & a>0 \\
\frac{4-\pi}{\pi}\left[a_{-\max }-\hat{a}(k)\right]^{2} & \alpha>0\end{cases}
\end{aligned}
$$

In formula (7), $a_{\max }$ is the max positive acceleration in $x$ axil, $a_{-\max }$ is $x$ the max negative acceleration in $x$ axil. We can calculate the variance and average acceleration in the same way.

Then we substitute the initial parameter for simulation. The initial parameters are as flow:

$$
\begin{aligned}
& x(0)=L_{x}(0), v_{x}(0)=0 \mathrm{~m} / \mathrm{s}, a_{x}=0 \mathrm{~m} / \mathrm{s}^{2} \\
& y(0)=L_{y}(0), v_{y}(0)=0 \mathrm{~m} / \mathrm{s}, a_{y}=0 \mathrm{~m} / \mathrm{s}^{2} \\
& \varepsilon_{x}(0)=\varepsilon_{y}(0)=0 \mathrm{~m}, P(0)=0 .
\end{aligned}
$$

Then we get the simulation results. Results of Kalman filter is shown in Figure 1 and 2, which respectively show the position error in $\mathrm{x}$ axil and y axil before and after the filter. From Figure 1 and Figure 2, we know that the error of the GPS positioning system is within 5 meters, the filtering effect is good, and the model is simple and the calculation is small.

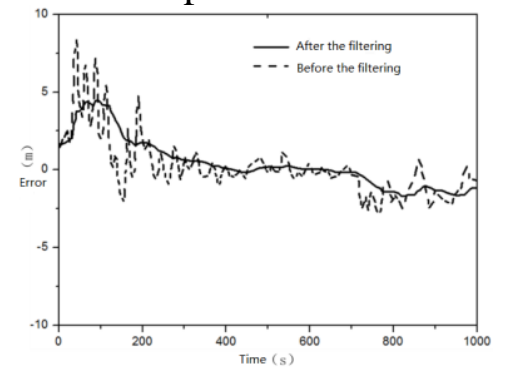

Figure 1. Eerror in $\mathrm{x}$ axil before and after filtering

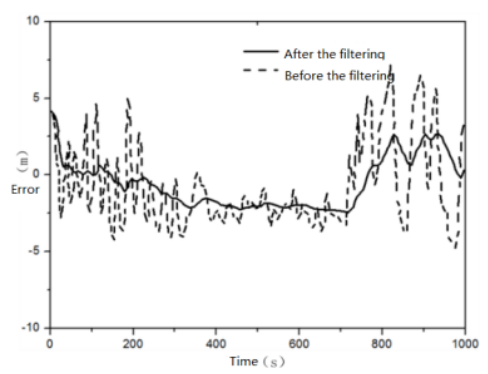

Figure 2. Error in y axil before and after filtering

\section{The recognition of ramp and curve based on the vehicle location and characteristics of line}

Here introduce vehicle lateral acceleration acquisition method. We assume that and are vertical and horizontal coordinates, is longitudinal velocity component, is lateral velocity component. So vertical acceleration and lateral acceleration are shown flow:

$$
\begin{aligned}
& a_{x}=\frac{\mathrm{d} u}{\mathrm{~d} t}-v \frac{\mathrm{d} \theta}{\mathrm{d} t}=\dot{u}-v \omega_{r} \\
& a_{y}=\dot{v}+u \omega_{r}
\end{aligned}
$$

We can calculate the differential equation of two degrees of freedom.

$$
\begin{aligned}
& k_{1}\left(\beta+\frac{a \omega_{r}}{u}-\delta\right)+k_{2}\left(\beta-\frac{b \omega_{r}}{u}\right)=m\left(\dot{v}+u \omega_{r}\right) \\
& a k_{1}\left(\beta+\frac{a \omega_{r}}{u}-\delta\right)-b k_{2}\left(\beta-\frac{b \omega_{r}}{u}\right)=I_{z} \dot{\omega}_{r}
\end{aligned}
$$

We get the differential equation of motion:

$$
\left\{\begin{array}{c}
\left(k_{1}+k_{2}\right) \beta+\frac{1}{u}\left(a k_{1}-b k_{2}\right) \omega_{r}-k_{1} \delta=m\left(\dot{v}+u \omega_{r}\right) \\
\left(a k_{1}-b k_{2}\right) \beta+\frac{1}{u}\left(a^{2} k_{1}+b^{2} k_{2}\right) \omega_{r}-a k_{1} \delta=I_{z} \dot{\omega}_{r}
\end{array}\right.
$$

Finally, we can get the computational formula of lateral acceleration: 


$$
a_{y}=\frac{\delta}{\frac{L}{u_{y}^{2}}+K L}
$$

We can calculate the resistance of the ramp through vehicle longitudinal dynamics theory to calculate the size of the ramp. The calculating formula is shown as flow:

$$
F_{i}=\frac{T_{m} i_{g} i_{0} \eta_{T}}{r}-G f-\frac{C_{D} A}{21.15} u_{a}^{2}-\delta m \frac{\mathrm{d} u}{\mathrm{~d} t}
$$

The ramp identification algorithm only needs to identify whether or not to enter the ramp condition, and we can simplify the ramp identification algorithm without external devices such as sensors.

\section{Comprehensive shift strategy of pure electric bus based on fixed line}

To achieve the control objective, a threshold control method is used based on the degree of urgency of the curve. The basic idea is to set an urgency degree threshold for entry and exit of corner, within the range of the threshold shift is forbidden, while out of corners to set a delay, in the delay range shift is also prohibited to ensure the stability of the pure electric bus out of the corner. After completion of the bend the normal shift schedule is restored. The amendatory shift schedule is shown in figure 3.

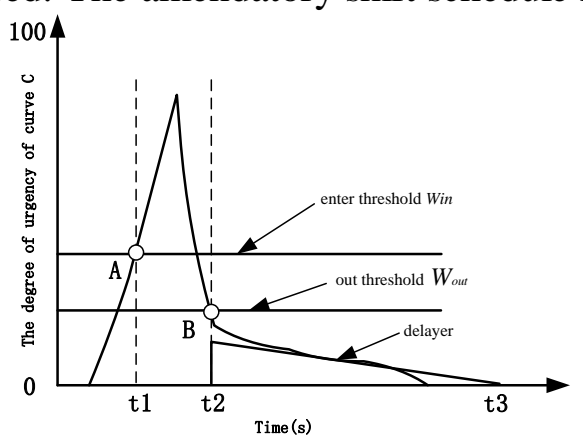

Figure 3. Gear modification in a curve

When $\mathrm{t}$ is in $0 \sim \mathrm{t} 1$, there is no gear modification;

When $\mathrm{t}$ is in $\mathrm{t} 1 \sim \mathrm{t} 2$, there is gear modification;

When $\mathrm{t}$ is in $\mathrm{t} 2 \sim \mathrm{t} 3$, trig delay timer, there is gear shift;

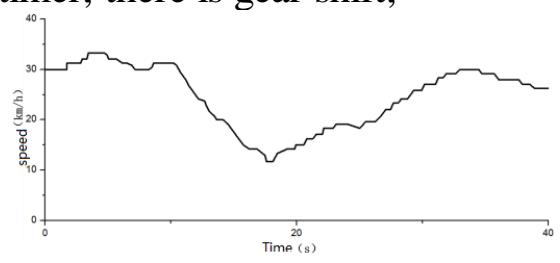

Figure 4. Speed changes in a curve

\section{Experimental verification}

When pure electric bus drive in a curve conditions, the speed changes are shown in figure 4 , and the lateral acceleration are shown in figure 5. The gear change which used the basic shift schedule and the amendatory shift schedule are shown in figure 6.From the figure 6. We can know that there is shift cycle in the basic shift schedule and there is not shift cycle in the amendatory shift schedule. Because the degree of urgency of test curve has reached a threshold, so shift is banned. The shift strategy solved the problem of shift cycle, and improved the comfort and safety of pure electric bus at a curve.
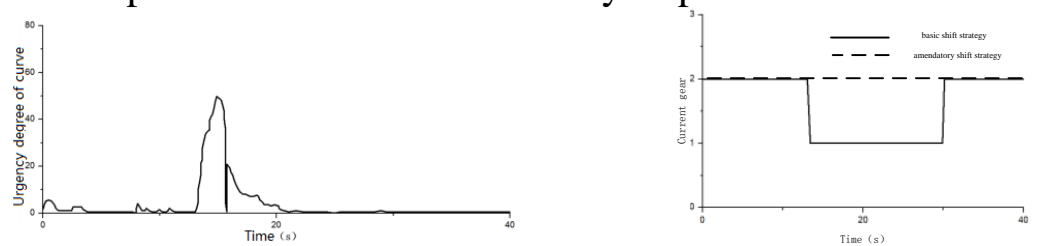

Figure 5. Lateral acceleration in a curve

Figure 6. Shift using basic and amendatory shift strategy 


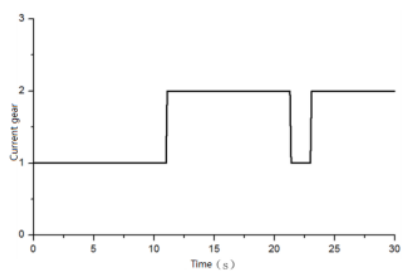

Figure 7. Gear shift using basic shift strategy

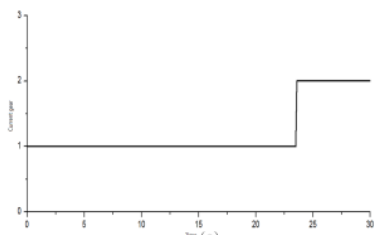

Figure 8. Gear shift using amendatory shift strategy

When using the basic shift schedule in the ramp, the shift change is shown in Figure 7. with the rise of the speed of a pure electric car reaches the upshift points, the car is promoted to second gear from the first gear, thereafter slope at a maximum the driving force in second gear is insufficient, resulting in decreased speed and the second gear down to first gear, then the driving force rises and the speed increases, which generates the ramp cycle shift problem. With ramp revised shift strategy pure electric passenger gear in the ramp change is shown in Figure 8. In the ramp, the vehicles delayed rise in second gear and hold this gear to the top of the ramp. In this way we can ensure the dynamic as well as solve the problem of circular shift to ensure the pure electric bus ramp driving stability and safety.

\section{Conclusion}

According to the characteristics of pure electric bus fixed-point and fixed line, we identify the position accurately in the various driving conditions, and developed a corresponding shift control strategy. The real vehicle experiments show that amendatory shift strategy can solve unexpected shift cycle phenomenon in curve and ramp, and improve driving safety and comfort of pure electric bus.

\section{Acknowledgments}

This work was supported by International S\&T Cooperation Program of China (Grant No. 2014DFA71790), the Key Scientific and Technological Project of Jilin Province (Grant No. 20160519008JH), Chinese National Natural Science Foundation(Grant No. 51575220) and Graduate Innovation Fund of Jilin University(Grant No. 2016170)”.

\section{References}

[1] Qin D, Zhou B, Hu M, Hu J, Wang X.2011. Parameter design of powertrain system of electric vehicle with two-speed gearbox. Journal of Chongqing University, 34(1):1-5.

[2]He Z, Bai H, Yang J.2006.Eliminating Strategy of AMT Vehicles Shift Hunting. Journal of agricultural machinery, 37 (7):9-13.

[3]Zhang Z, Zhou G. 2012. Farm Machinery on-board GPS Location Accuracy Simulation and Research. Computer Science, 39(12):216-219.

[4]Li S. A Research on Comprehensive Shift Schedule for Pure Electric Bus. Master graduation thesis of Jilin University.

[5]Chen Y, Liang W, Xi J.2011.A Study on the Control Strategy for the Gear Shifting of AMT in a Electric Bus. Automotive Engineering, 33(5):405-410.

[6]Guo M, Nan J, Guo F. 2013. Development of Vehicle Control Unit for an Extended-Range Electric Bus. Journal of Chongqing University of Technology, 27(1):7-12. 